\title{
Fitoconstituintes isolados da fração em diclorometano das folhas de Vernonia tweediana Baker
}

\author{
Ricardo Basso Zanon, ${ }^{1}$ Danielle Fontana Pereira, ${ }^{1}$ Ticiane Krapf Boschetti, ${ }^{1}$ Mariane dos \\ Santos, ${ }^{1}$ Margareth Linde Athayde ${ }^{*, 2}$ \\ ${ }^{1}$ Laboratório de Fitoquímica, Departamento de Farmácia Industrial, Universidade Federal de Santa Maria, \\ Campus Camobi, 97119-900 Santa Maria-RS, Brasil, \\ ${ }^{2}$ Departamento de Farmácia Industrial, Universidade Federal de Santa Maria, Campus Camobi, 97119-900 \\ Santa Maria-RS, Brasil
}

\begin{abstract}
RESUMO: Fracionamento cromatográfico da fração em diclorometano obtida do extrato etanólico das folhas de Vernonia tweediana Baker (Asteraceae) conduziu ao isolamento de $\alpha$-amirina, $\beta$-amirina, lupeol, $\beta$-sitosterol, estigmasterol e espinasterol. As estruturas foram identificadas através de técnicas espectroscópicas usuais, além da comparação com dados relatados na literatura. Os compostos isolados são relatados pela primeira vez para a espécie $V$. tweediana.
\end{abstract}

Unitermos: Vernonia tweediana, Asteraceae, triterpenos, esteróides.

\begin{abstract}
Phytoconstituents isolated from dichloromethane fraction of Vernonia tweediana Baker leaves". Fractionation of the dichloromethane -soluble fraction from the ethanol extract of the leaves of Vernonia tweediana (Asteraceae) led to the isolation of $\alpha$-amyrin, $\beta$-amyrin, lupeol, $\beta$-sitosterol, stigmasterol and spinasterol. The structures of the isolates were elucidated by spectroscopic analysis and comparison with literature data. The isolated compounds are reported for the first time to the species $V$. tweediana.
\end{abstract}

Keywords: Vernonia tweediana, Asteraceae, triterpenes, steroids.

\section{INTRODUÇ̃̃O}

O gênero Vernonia (Asteraceae) abrange uma grande diversidade de espécies, com representantes no mundo inteiro. As espécies deste gênero são utilizadas em várias partes do mundo para o tratamento de diversas enfermidades, salientando-se o uso para o tratamento da malária, em doenças respiratórias e distúrbios gastrintestinais. Os principais constituintes relatados para o gênero são lactonas sesquiterpênicas, flavonóides, triterpenos, esteróides, lignóides e carotenóides, além de alcalóides e taninos (Awe et al., 1998; Carvalho et al., 1999; Barbosa-Filho et al., 2008).

No Brasil, várias espécies de Vernonia são utilizadas empiricamente pela população, destacandose o macerado de folhas de $V$. condensata Baker, usado como analgésico, anti-ulcerogênico e para a prevenção de distúrbios no estômago e fígado (Frutuoso et al., 1994) e os extratos alcoólicos de V. scorpioides (Lam.) Pers., utilizados no tratamento dos mais variados ferimentos cutâneos (Dalazen et al., 2005).

A espécie Vernonia tweediana Baker, conhecida como "assa-peixe", é um arbusto e representa uma das principais plantas invasoras de pastagens artificiais implantadas ao longo da costa atlântica (Cabrera \& Klein, 1980). Característica da região sul do Brasil, Paraguai e Argentina, a planta é popularmente utilizada para o tratamento de doenças respiratórias, constituindo-se de uma alternativa de tratamento em gripes, bronquites e tosses. A ausência de estudos sobre a espécie e a importância do registro químico de espécies endêmicas dos biomas brasileiros, principalmente as que são utilizadas para fins terapêuticos, motivaram o presente trabalho, que descreve os primeiros resultados obtidos no estudo fitoquímico da fração em diclorometano das folhas de $V$. tweediana.

\section{MATERIAL E MÉTODOS}

\section{Instrumentação e procedimentos gerais}

Os espectros de absorção na região do infravermelho (IV) foram registrados em espectrômetro Nicolet Magna 550, utilizando-se pastilhas de KBr. Os espectros de Ressonância Magnética Nuclear, RMN de ${ }^{1} \mathrm{H}(400 \mathrm{MHz})$ e de ${ }^{13} \mathrm{C}(100 \mathrm{MHz})$, foram realizados em espectrômetro Brucker DPX, utilizando-se $\mathrm{CDCl}_{3}$ como solvente e TMS como padrão de referência interna.As análises de Cromatografia Gasosa acoplada ao Espectrômetro de Massas (CG-EM) foram realizadas em cromatógrafo Hewlett-Packard 6890 Series Plus +, equipado com injetor automático split-splitless modelo 
HP 6890 Series GC Auto Sampler Controller e detector seletivo de massas modelo HP 5973 MSD, utilizando-se coluna capilar cromatográfica de sílica fundida HP-5 MS (30 m x 0,32 mm diâmetro interno e espessura do filme $0,25 \mu \mathrm{M})$ com $5 \%$ fenil e $95 \%$ de metilsiloxano.O gás de arraste foi hélio (fluxo de $2 \mathrm{~mL} / \mathrm{min}$ ). Temperatura do injetor de $250{ }^{\circ} \mathrm{C}$ com programação de taxa de aquecimento de $12{ }^{\circ} \mathrm{C} / \mathrm{min}$ até $280{ }^{\circ} \mathrm{C}$. Energia de ionização $70 \mathrm{eV}$. Para as cromatografias em coluna, utilizou-se gel de sílica 60 da Merck, com tamanho de partícula 0,063-0,200 mm (70-230 mesh-ASTM). Para cromatografia de camada delgada (CCD) utilizou-se cromatofolhas de gel de sílica $\mathrm{GF}_{254}$ (Merck) e gel de sílica impregnado com solução de nitrato de prata a $10 \%$ (Stahl, 1969). Para a detecção das substâncias, utilizouse anisaldeído-sulfúrico seguido de aquecimento.

\section{Material vegetal}

Folhas de $V$. tweediana foram coletadas em abril de 2004 em Ijuí-RS e identificadas pelo Dr. Geraldo Ceni Coelho do Departamento de Biologia e Química (DeBQ/UNIJUÍ). A exsicata foi depositada no Herbário do Departamento de Biologia da UFSM, catalogada sob o número SMDB 9536.

\section{Extração e isolamento dos constituintes}

As folhas foram secas a temperatura ambiente e moídas, resultando em uma massa de $1,9 \mathrm{Kg}$ de material vegetal, que foi submetido à maceração em EtOH $65 \%$ em água (v/v), na proporção de $15 \mathrm{~g} / 100$ $\mathrm{mL}$ de solvente, durante sete dias, com agitação diária. $\mathrm{O}$ extrato obtido foi filtrado e o EtOH removido sob pressão reduzida em evaporador rotatório. $\mathrm{O}$ extrato aquoso remanescente foi fracionado com solventes de polaridade crescente $\left(\mathrm{CH}_{2} \mathrm{Cl}_{2}\right.$, AcOEt e $n$ - $\mathrm{BuOH}$ e $3 \mathrm{x}$ $200 \mathrm{~mL}$ para cada solvente). A fração em $\mathrm{CH}_{2} \mathrm{Cl}_{2}(10,0$ g) foi submetida à cromatografia em coluna "flash" sobre gel de sílica $60(225 \mathrm{~g})$ usando-se inicialmente como fase móvel apenas $\mathrm{CH}_{2} \mathrm{Cl}_{2}(1 \mathrm{~L})$ e posteriormente as misturas de $\mathrm{CH}_{2} \mathrm{Cl}_{2}: \mathrm{EtOH} 9: 1 \mathrm{v} / \mathrm{v}(1 \mathrm{~L})$ e $8,5: 1,5 \mathrm{v} / \mathrm{v}$ (1L). Foram coletadas 30 frações de $\pm 100 \mathrm{~mL}$, que, após monitoramento por cromatografia em camada delgada (CCD), foram agrupadas com base na semelhança de perfil cromatográfico. O agrupamento de frações conduziu à obtenção de duas principais sub-frações, codificadas como I $(2,082 \mathrm{~g})$ e II $(131 \mathrm{mg})$. Parte da sub- fração I $(600 \mathrm{mg})$ foi cromatografada em coluna de gel de sílica $60(58 \mathrm{~g})$ e eluída com $\mathrm{CH}_{2} \mathrm{Cl}_{2}$ éter de petróleo $(9: 1, \mathrm{v} / \mathrm{v})$, obtendo-se outra sub-fração (230 $\mathrm{mg}$ ) que foi novamente submetida à cromatografia em coluna de $\mathrm{SiO}_{2}-\mathrm{AgNO}_{3}(22 \mathrm{~g})$, originando o composto $1(43,6 \mathrm{mg})$ e a mistura $\mathbf{2}+\mathbf{3}(50 \mathrm{mg})$.A sub-fração II $(131 \mathrm{mg})$ foi submetida a cromatografia em gel de sílica 60 (40 g), tendo como eluente $\mathrm{CH}_{2} \mathrm{Cl}_{2}$ :éter de petróleo $(7: 3, v / v)$. Após monitoramento por cromatografia em camada delgada, as frações foram agrupadas com base na semelhança de perfil cromatográfico. Frações reunidas $(61 \mathrm{mg})$ foram novamente cromatografadas em coluna de $\mathrm{SiO}_{2}-\mathrm{AgNO}_{3}(12 \mathrm{~g})$. As frações obtidas foram analisadas por CCD $\left(\mathrm{SiO}_{2} / \mathrm{AgNO}_{3}\right)$ verificandose a separação da substância $4(7 \mathrm{mg})$ e a da mistura composta por $5+6(27 \mathrm{mg})$.

\section{RESULTADOS E DISCUSSÃO}

Sucessivos fracionamentos cromatográficos da fração em $\mathrm{CH}_{2} \mathrm{Cl}_{2}$ obtida a partir do extrato hidroetanólico $(65 \%)$ conduziram ao isolamento de três triterpenos e três fitoesteróides (Figura 1), cujas estruturas foram identificadas com base na análise de dados de IV, EM e RMN, bem como pela comparação com dados descritos na literatura (Ogunkoya, 1981; Chaturvedula et al., 2004; Olea \& Roque 1990; DeEknankul \& Potduang, 2003; Forgo \& Kövér, 2004; Villaseñor et al.,1996; Cortez et al., 2006).

$\mathrm{O}$ espectro de $\mathrm{RMN}$ de ${ }^{13} \mathrm{C}$ de $\mathbf{1}$, indicou a presença de trinta átomos de carbono. Desses sinais, dois referentes a carbono $\mathrm{sp}^{2}$, são característicos de ligação dupla em compostos com esqueleto lupeno $\left(\delta_{\mathrm{C}}\right.$ 109,3 e $150,9 \mathrm{ppm}$ ), que conjuntamente com o grupo metil em $\delta_{C} 19,3$, são indicativos de grupo isopropenil (C-29, C-20 e C-30, respectivamente). No espectro de $\mathrm{RMN}$ de ${ }^{1} \mathrm{H}$, dois dubletos em $\delta_{\mathrm{H}} 4,56$ e 4,68 correspondem aos dois hidrogênios geminais olefínicos (H-29a e H-29b). Estes sinais, somados ao $\delta_{H}$ do sinal de metila em 1,67 ppm, corroboram com a indicação da presença de isopropenil, comprovando assim que $\mathbf{1}$ trata-se de um triterpeno pentacíclico do tipo lupeno (Chaturvedula et al., 2004; Aguiar et al., 2005). Na análise por CG da substância 1 foi possível observar um único pico com tempo de retenção de $26,08 \mathrm{~min}$. O EM (IE) mostrou pico em $\mathrm{m} / \mathrm{z} 426$, que corresponde ao pico do íon molecular de 1. Outros picos oriundos da fragmentação do tipo Retro-Diels-Alder do anel C, são típicos de triterpenos pentacíclicos, principalmente os de maior intensidade como os m/z 218; 207; 203 e 189. Destes, os mais intensos foram m/z 189 e 207, que são característicos de fragmentações de compostos do tipo lupeno (Ogunkoya, 1981), permitindo a identificação do composto 1 como lupeol.

A elucidação da mistura $\mathbf{2}+\mathbf{3}$ ( $\alpha$ e $\beta$-amirina, respectivamente), foi efetuada com base nos assinalamentos de $\mathrm{RMN}$ de ${ }^{13} \mathrm{C}$ e $\mathrm{RMN}$ de ${ }^{1} \mathrm{H}$ descritos na literatura (Ogunkoya, 1981; Olea \& Roque 1990; Baykal et al.,1998; Bandeira et al., 2007). O espectro de $\mathrm{RMN}$ de ${ }^{13} \mathrm{C}$, indicou a presença de 56 átomos de carbonos, sugerindo uma mistura binária de triterpenos. Observaram-se sinais que caracterizam as séries urseno (139,2 e 124,4 ppm) e oleaneno (121,7 e 145,2 ppm). No cromatograma obtido por CG-EM foi possível observar dois picos em tempos de retenção de 25,28 ( $\beta$-amirina) e 26,07 ( $\alpha$-amirina) min., sendo as proporções 
<smiles>C=C(C)C1CCC2(C)CC[C@]3(C)C(CCC4[C@@]5(C)CCC(O)C(C)(C)C5CC[C@]43C)C12</smiles>

1

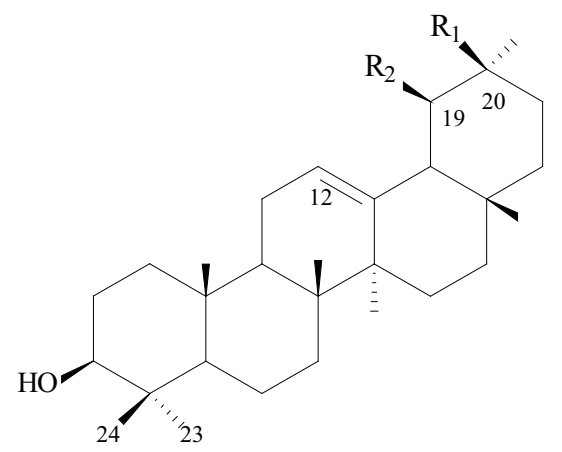

$2 \mathrm{R}_{1}=\mathrm{H} \quad \mathrm{R}_{2}=\mathrm{CH}_{3}$

$3 \mathrm{R}_{1}=\mathrm{CH}_{3} \quad \mathrm{R}_{2}=\mathrm{H}$

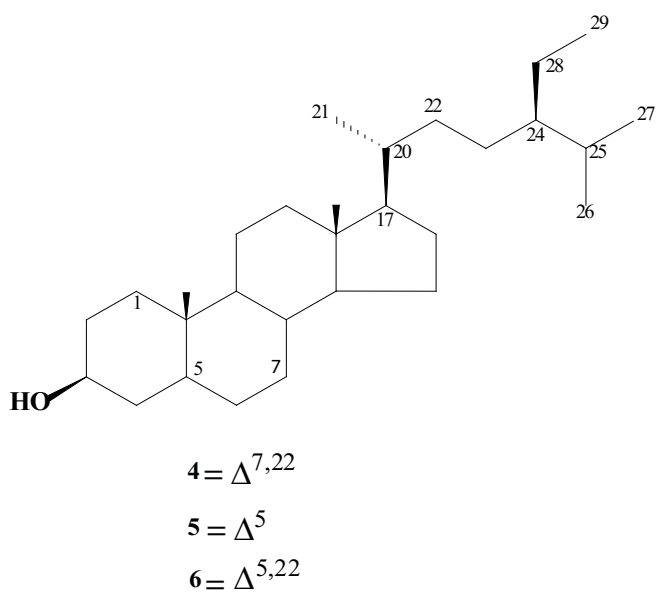

Figura 1. Compostos isolados de V. tweediana.

entre eles 1:1 (verificadas pelas áreas dos picos). Os espectros de massas foram muito semelhantes, diferindo apenas na intensidade de alguns picos, principalmente o pico em $m / z 203\left(m / z 218-\mathrm{CH}_{3}\right.$, mais intenso $\beta$-amirina). Ambos mostraram picos em $m / z$ 426, correspondentes aos íons moleculares dos dois isômeros. Picos oriundos da fragmentação do tipo Retro-Diels-Alder do anel C, típicos de olean-12-enos e ursan-12-enos, foram observados em $\mathrm{m} / \mathrm{z}$ 218; 203 e 189 (Ogunkoya, 1981; Morita et al., 2000).

$\mathrm{O}$ espectro de RMN de ${ }^{13} \mathrm{C}$ de $\mathbf{5}+\mathbf{6}$ indicou a presença de 43 átomos de carbonos, sugerindo uma mistura binária. Quatro sinais na região de carbonos $\mathrm{sp}^{2}$ $(121,7,129,3,138,3$ e 140,8 ppm) indicam duas ligações duplas. Os sinais em $\delta$ c 121,7 e 140,8 são característicos de esteróides com uma ligação dupla entre C-5 e C-6 (Forgo \& Kövér, 2004;De-Eknankul \& Potduang, 2003; Tomaz et al., 2008) e os sinais em $\delta c 129,3$ e 138,3 são característicos de uma ligação dupla entre C-22 e C-23 (Forgo; Kövér, 2004). O $\beta$-sitosterol (24 $\alpha$-etil-colestan-

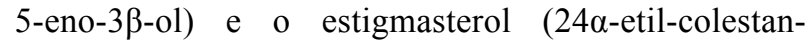
5,22-dieno-3 $\beta$-ol) possuem uma mesma insaturação $\left(\Delta^{5}\right)$, portanto, os sinais nos espectros de RMN de ${ }^{13} \mathrm{C}$ irão coincidir e assim C-5 e C-6 estarão mais intensos quando comparados com os outros dois sinais referentes a insaturação em $\Delta^{22}$, presente somente em estigmasterol (De-Eknankul \& Potduang, 2003; Ayres et al., 2008). No espectro de RMN de ${ }^{1} \mathrm{H}$ destacam-se o singlete largo em $\delta_{\mathrm{H}} 5,36(\mathrm{H}-6)$, comum em ambos os compostos e os duplo-dubletes em $\delta_{\mathrm{H}} 5,02(\mathrm{~J}=8$ e 16 $\mathrm{Hz}, \mathrm{H}-23)$ e $5,16(\mathrm{~J}=8$ e $15 \mathrm{~Hz}, \mathrm{H}-22)$, que caracterizam os demais hidrogênios olefínicos do estigmasterol (DeEknankul \& Potduang, 2003; Forgo \& Kövér, 2004). O cromatograma resultante do CG-EM mostrou os dois picos referentes ao estigmasterol ( $\mathrm{TR}=23,83 \mathrm{~min}$.) e ao $\beta$-sitosterol ( $\mathrm{TR}=24,70 \mathrm{~min}$.). As concentrações percentuais, com base nas áreas dos picos, foram de 91:9 estigmasterol (6)/ $\beta$-sitosterol (5).

$\mathrm{O}$ espectro de $\mathrm{RMN}$ de ${ }^{13} \mathrm{C}$ de 4 registrou a presença de 29 átomos de carbono. Desses sinais, quatro característicos de carbonos $\mathrm{sp}^{2}\left(\delta_{\mathrm{C}} 117,5,129,5,138,1\right.$ e $140,8 \mathrm{ppm}$ ) sugerem a presença de duas ligações duplas. Estes sinais são característicos de compostos com esqueletos esteroidais ramificados, quando uma das insaturações está localizada entre C-7 e C-8 $\left(\delta_{\mathrm{C}}\right.$ $117,5$ e 138,1$)$ e a outra entre C-22 e C-23 $\left(\delta_{C} 129,5\right.$ e 140,8) (Villaseñor et al.,1996; Gomes \& Alegrio, 1998). O EM mostrou pico em $\mathrm{m} / \mathrm{z} 412$, indicando a formula molecular $\mathrm{C}_{29} \mathrm{H}_{50} \mathrm{O}$. O pico base a m/z 271 caracteriza a presença de ligação dupla na posição 7 em moléculas esteroidais (Villaseñor et al.,1996).O espectro de IV apresenta um sinal em $970 \mathrm{~cm}^{-1}$, indicativo da posição trans em $\mathrm{HC}=\mathrm{CH}$ (C22 e C23) (Villaseñor et al.,1996). $\mathrm{O}$ singleto largo em $\delta_{\mathrm{H}} 5,09$ foi atribuído ao hidrogênio olefínico H-7 e os sinais em $\delta_{\mathrm{H}} 4,96(1 \mathrm{H}, d d, \mathrm{~J}=8$ e 15 $\mathrm{Hz}, \mathrm{H}-22$ ou H-23) e $\delta_{\mathrm{H}} 5,12(1 \mathrm{H}, d d, \mathrm{~J}=8$ e $15 \mathrm{~Hz}$, $\mathrm{H}-22$ ou H-23) aos dois hidrogênios olefínicos restantes. A comparação dos deslocamentos químicos de RMN de ${ }^{13} \mathrm{C}$ e RMN de ${ }^{1} \mathrm{H}$ da substância 4, com os registrados na literatura (Garg \& Nes, 1984a,b; Villaseñor et al., 1996; Gomes \& Alegrio, 1998; Paulo et al., 2005) permitiu propor a estrutura do espinasterol.

Todos os compostos isolados foram descritos previamente para o gênero (Bohlmann \& Zdero, 1982; Misra et al., 1984). Estas informações sobre a espécie 
Vernonia tweediana Baker, conhecida como pertencente a um grupo de plantas de ocorrência marcante na América do Sul, apresentam os primeiros resultados do estudo fitoquímico da fração em diclorometano das folhas da planta. No entanto, o potencial químico merece ser ampliado e a eficácia biológica avaliada.

\section{AGRADECIMENTOS}

À CAPES pela concessão da bolsa de Mestrado (R.B.Z.), ao CNPq (BIC de T. K. B.), ao FIEX/UFSM (Bolsa de Extensão de M.S.), ao Departamento de Química da UFSM, pela confecção dos espectros de RMN e ao Dr. Geraldo Ceni Coelho (UNIJUÍ) pela identificação do material botânico.

\section{REFERÊNCIAS}

Aguiar RM, David JP, David JM 2005. Unusual naphthoquinones, catechin and triterpene from Byrsonima microphilla. Phytochemistry 66: 2388-2392.

Awe SO, Olajide O, Makinde JM 1998. Cathartic effect of the leaf extract of Vernonia amygdalina. Phytother Res 12: $57-58$.

Ayres MCC, Brandão MS, Vieira-Júnior GM, Menor JCAS, Silva HB, Soares MJS, Chaves MH 2008. Atividade antibacteriana de plantas úteis e constituintes químicos da raiz de Copernicia prunifera. Rev Bras Farmacogn 18: 90-97.

Bandeira PN, Lemos TLG, Costa SMO, Santos HS 2007. Obtenção de derivados da mistura triterpenoídica $\alpha$ e $\beta$-amirina. Rev Bras Farmacogn 17: 204-208.

Barbosa-Filho JM, Alencar AA, Nunes XP, Tomaz, ACA, Sena-Filho JG, Athayde-Filho PF, Silva MS, Souza MFV, Cunha EVL 2008. Sources of alpha-, beta-, gamma-, delta- and epsilon-carotenes: A twentieth century review. Rev Bras Farmacogn 18: 135-154.

Baykal T, Panayr T, Tasdemir D, Sticher O, Çalis I 1998. Triterpene saponins from Scabiosa rotata. Phytochemistry 48: 867-873.

Bohlmann F, Zdero C 1982. Allenic germacranolides, bourbonene derived lactones and other constituents from Vernonia species. Phytochemistry 20: 473-480.

Cabrera AL, Klein RM 1980. Fascículo Compostas: 3. Tribo: Vernoniae. Flora Ilustrada Catarinense 324-326.

Carvalho MG, Costa PM, Abreu HS 1999. Flavones from Vernonia diffusa. J Braz Chem Soc 10: 163-166.

Chaturvedula VSP, Zhou B, Gao Z, Thomas SJ, Hecht SM, Kingston DGI 2004. New lupane triterpenoids from Solidago canadensis that inhibit the lyase activity of DNA polymerase $\beta$. Bioorg Med Chem 12: 6271-6275.

Cortez LER, Cortez DAG, Ferreira AG, Vieira PC, Silva MFGF, Fernandes JB 2006. Constituintes químicos de Almeidea coerulea (Nees \& Mart.) A. St.-Hil. Rutaceae. Rev Bras Farmacogn 16: 164-169.

Dalazen P, Molon A, Biavatti MW, Kreuger MRO 2005. Effects of the topical application of the extract of Vernonia scorpioides on excisional wounds in mice. Rev Bras Farmacogn 15: 82-87.
De-Eknankul W, Potduang B 2003. Biosynthesis of $\beta$-sitosterol and stigmasterol in Croton sublyratus proceeds via a mixed origin of isoprene units. Phytochemistry 62: 389-398.

Forgo P, Kövér K 2004. Gradient enhanced selective experiments in the ${ }^{1} \mathrm{H}$ NMR chemical shift assignment of the skeleton and side-chain ressonances of stigmasterol, a phytosterol derivative. Steroids 69 : 43-50.

Frutuoso VS, Gurjão MRR, Cordeiro RSB, Martins MA 1994. Analgesic and anti-ulcerogenic effects of a polar extract from leaves of Vernonia condensata. Planta Med 60: 21-25.

Garg VK, Nes WR 1984a. Studies on the C-24 configurations of $\Delta^{7}$-sterols in the seeds of Cucurbita maxima. Phytochemistry 23: 2919-2924.

Garg VK, Nes WR 1984b. Codisterol and other $\Delta^{5}$-sterols in the seeds of Cucurbita maxima. Phytochemistry 23: 2925-2929.

Gomes DCF, Alegrio LV 1998. Acyl steryl glycosides from Pithecellobium cauliflorum. Phytochemistry 49: 1365-1367.

Misra TN, Singh RS, Upadhyay J, Srivastava R 1984. Chemical constituents of Vernonia cinerea. Isolation and estructure elucidation of a new pentacyclic triterpenoid. J Nat Prod 47: 865-867.

Morita M, Shibuya M, Kushiro T, Masuda K, Ebizuka, Y 2000. Molecular cloning and functional expression of triterpene synthases from pea (Pisum sativum). New $\alpha$-amyrin-producing enzyme is a multifunctional triterpene synthase. Eur J Biochem 267: 3453-3460.

Ogunkoya L 1981. Aplication of mass spectrometry in structural problems in triterpenes. Phytochemistry 20: 121-126.

Olea RSG, Roque NF 1990. Análise de misturas de triterpenos. Quim Nova 13: 278-281.

Paulo A, Dias C, Jimeno ML, Borges C, Nascimento JA 2005. A new $\Delta^{7,22}$ sterol from the bulbs of Autonöe madeirensis. Fitoterapia 76: 765-767.

Stahl E 1969. Thin-layer chromatography - a laboratory handbook. $2^{\mathrm{a}}$ ed. Springer-Verlag: Berlin, Heidelberg, New York.

Tomaz ACA, Nogueira RBSS, Pinto DS, Agra MF, Souza MFV, Cunha EVL 2008. Chemical constiuents from Richardia grandiflora (Cham. \& Schltdl.) Steud. (Rubiaceae). Rev Bras Farmacogn 18: 47-52.

Villaseñor IM, Lemon P, Palileo A, Bremner JB 1996. Antigenotoxic spinasterol from Cucurbita maxima flowers. Mutat Res 360: 89-93. 